MODELING, IDENTIFICATION AND CONTROL, 2001, vOL. 22, NO. 3, 141-152

doi:10.4173/mic.2001.3.2

\title{
Control of a Gravity Gradient Stabilised Satellite using Fuzzy Logic
}

\author{
AAGE SKULLESTAD, KJETIL OLSEN, \\ STEIN RENNEHVAMMEN and HAAVARD FLOYSTAD
}

Keywords: 3-axes stabilisation, satellite, Fuzzy control, simulation.

\begin{abstract}
This paper describes attitude control of a small gravity gradient stabilised satellite. A gravity gradient stabilised satellite has limited stability and pointing capabilities, and magnetic coils are added in order to improve the accuracy of the attitude control. The magnetic coils are controlled using a fuzzy logic controller, based on a combination of membership functions and rules. The control of the pitch axis is separated from the roll and azimuth axes and excellent pitch angle accuracy is achieved. The roll and azimuth axes are controlled using a common magnetic coil, that has a non-linear and time-varying torque characteristic and, therefore, accurate roll and azimuth angular control become much more difficult to achieve. However, combining one roll controller and two azimuth controllers result in an acceptable roll and azimuth angular accuracy after a few orbital periods.
\end{abstract}

\section{Introduction}

This paper describes attitude control of a small satellite. The satellite is intended to fly in a low earth orbit at $800-1200 \mathrm{~km}$ altitude and its mission is to perform scientific measurements. The satellite is expected to be box-shaped, have a total mass of approximately $80 \mathrm{~kg}$ and be gravity gradient stabilised. A proposed $6 \mathrm{~m}$ long gravity boom, including a tip mass of $2 \mathrm{~kg}$, will play a key role in the gravity gradient stabilisation. The satellite is to be three-axis stabilised with its boom pointing Thewards.

Generally, a gravity gradient stabilised system has limited stability and pointing capability, and reaction wheels and/or magnetic coils are added in order to improve the attitude control. The best attitude accuracy is achieved using reaction wheels, but wheels increase the complexity and cost of the satellite. Magnetic coils allow cheaper satellites, and are an attractive solution to small, inexpensive satellites in low earth orbits.

The satellite will during separation from the launch vehicle be exposed to forces from the release mechanism and tumbling may occur. A detumbling mode is activated in order to calm down the movements. The gravitation boom will be deployed first when the movement of the satellite is sufficiently small. This paper deals with attitude control after the detumbling mode has successfully been completed and the boom is fully deployed. At this time all angles are assumed to be small and a linearised mathematical model of the satellite can be justified and is also applied in this paper. All simulations are performed using MATLAB.

Gravity gradient stabilisation has been used in attitude control since the early sixties (Hughes, 1986). Gravity gradient stabilisation combined with magnetic torquing, has gained increased attention as an attractive attitude control system (ACS) for small cheap satellites and is also proposed used in this satellite. Magnetic coils,

Aage Skullestad, Kongsberg Defence \& Aerospace, Kongsberg, Norway. Kjetil Olsen, Stein Rennehvammen, Haavard Floystad, Buskerud College of Engineering, Kongsberg, Norway 
mounted in the $\mathrm{x}, \mathrm{y}$ and $\mathrm{z}$ facets of the satellite main body, perform the magnetic torquing. The coils interact with the magnetic field of the earth and produce a satellite torque, which is used to control the rotation of the satellite. A problem is that both the direction and the strength of the geomagnetic field change and magnetic control become non-linear and time dependent.

Musser et al. (1989) combined gravity gradient stabilisation and magnetic coils and also showed that linear quadratic (LQ) control may be used to obtain three-axis stabilisation of a satellite. Bak et al. (1996) and Wisniewski (1996) all describe attitude control combining gravity gradient stabilisation, magnetic torquing and LQ control. This idea is also treated in Narheim et al. (1994), Cavallo et al. (1993) apply two magnetic coils and one reaction wheel controlled by a sliding mode strategy. This paper describes control of a gradient stabilised satellite using fuzzy logic.

Section 2 gives a short description of fuzzy logic. Section 3 presents a mathematical model of the satellite. Section 4 shows how the present low orbit satellite can be stabilised using gravity gradient stabilisation combined with coil control. Conclusions are found in section 5 .

\section{Fuzzy Logic}

Fuzzy logic was introduced by Zadeh in 1965, whereas the first practical fuzzy logic controllers were first implemented in the mid-seventies. Fuzzy control often applies to systems that cannot be accurately described by differential/difference equation framework. A complete fuzzy control system is shown in Figure 1.

The fuzzy controller consists of a fuzzy processor interfaced a pre and a postprocessor. The pre-processor allows pre-processing of the error signal, i.e. integral and derivative actions, filtering etc. The pre-processor often, in the case of integral actions, executes anti-windup. The fuzzy processor involves four main components, namely the fuzzification interface, the rule-base, the inference mechanism and the defuzzification interface. A brief explanation of these four main components is given below, a more detailed description can be found in an ordinary text book (Passino et al., 1998). The rule-base holds the knowledge, in the form of a set of rules. The rule base contains a large number of rules of the type "if-then". The inference mechanism evaluates which control rules are relevant at the current time and then decides what the input to the process should be. The inference engine applies the rule base and the input data from the pre-processor to draw a conclusion, which is the fuzzy output of the controller or the input to the defuzzification. The fuzzification modifies the inputs from the pre-processor so that they can be interpreted and

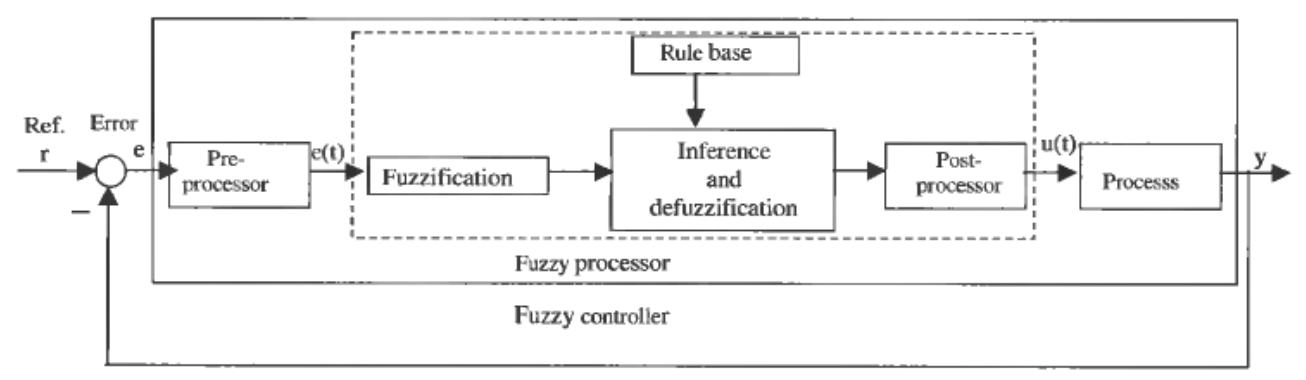

Figure 1. A complete fuzzy logic control system. 


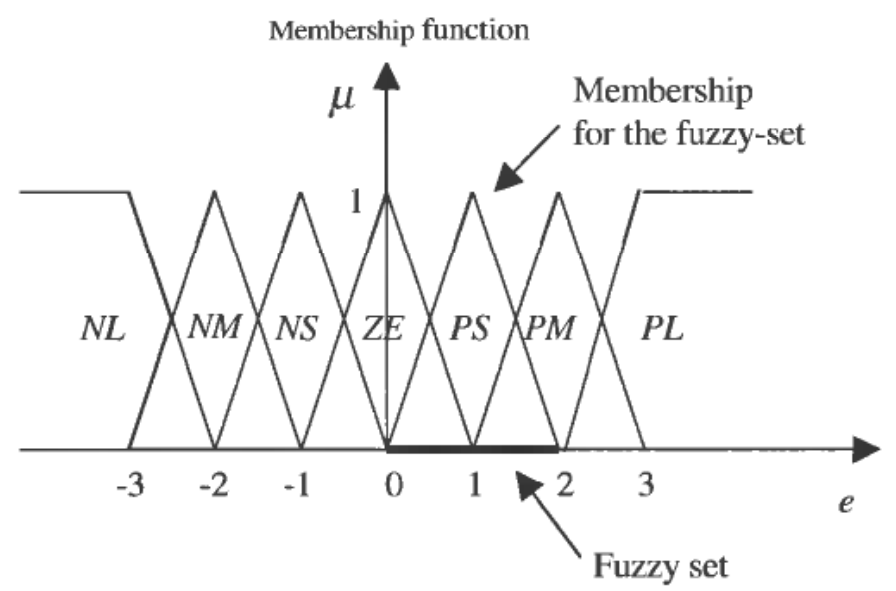

Figure 2. Graphical representation of an overlapping membership function.

compared to the rules in the rule-base. The fuzzification procedure simply consists of finding appropriate membership functions to the input data. Figure 2 shows an example of an overlapping membership function. $\mu$ is the grade of membership. The defuzzification interface converts the conclusion reached by the inference mechanism into the inputs of the process.

\section{Mathematical Model of the Satellite}

The satellite consists of a gravity boom connected to a satellite body. The gravity boom has a tip mass of $2 \mathrm{~kg}$ to improve the gravity gradient stabilisation. The gravity boom is flexible, but is in this paper, as a first order approximation considered to be rigid. Electromagnetic coils are added to improve both the three-axis stabilisation and the pointing properties. The attitude control is performed after the detumbling phase has ended and at that time the attitude angles can be regarded as small, and the non-linear mathematical satellite model can be linearised. The linearised model is modelled using MATLAB and several simulations are performed. The torque equation, assuming the satellite to be a stiff body, may be written (Skullestad, 1995),

$$
J^{B} \dot{\omega}_{B / I}+{ }^{B} \omega_{B / I} \times\left(J^{B} \omega_{B / I}\right)={ }^{B} T
$$

where:

$J=$ moment of inertia matrix referred to body frame, i.e. $J=\operatorname{diag}\left(I_{x}, I_{y}, I_{z}\right)$

${ }^{B} T=$ total torque acting on the satellite expressed in body frame components

${ }^{B} \omega_{B_{\mid} I}=$ angular velocity of body frame referred to inertial reference frame expressed in body frame components

The body frame aligns the principal-axis of the rigid body, hence, the moment of inertia matrix is simplified to a diagonal inertia matrix, (1). The external torque consists of the gravity gradient torque, coil torque, and the disturbance torque, and may be expressed as

$$
{ }^{B} T={ }^{B} T_{G}+{ }^{B} T_{A}+{ }^{B} T_{D}
$$

${ }^{B} T_{G}=$ gravity gradient torque expressed in body frame components and defined in chapter 3.1 
${ }^{B} T_{A}=$ magnetic coil torque expressed in body frame components and defined in chapter 3.2.

${ }^{B} T_{D}=$ disturbance torque expressed in body frame components.

(1) may be expanded in component form and expressed in body frame components (along the principal body axes) as

$$
\begin{aligned}
& I_{x} \dot{\omega}_{x}=\left(I_{y}-I_{z}\right) \omega_{y} \omega_{z}+T_{x} \\
& I_{y} \dot{\omega}_{y}=\left(I_{z}-I_{x}\right) \omega_{x} \omega_{z}+T_{y} \\
& I_{z} \dot{\omega}_{z}=\left(I_{x}-I_{y}\right) \omega_{x} \omega_{y}+T_{z}
\end{aligned}
$$

where: $\omega_{x}, \omega_{y}, \omega_{z}$ are the angular velocities of body frame, expressed in body frame components. $I_{x}, I_{y}, I_{z}$ are the moment of inertia in body frame and $T_{x}, T_{y}, T_{z}$ are the additional torques expressed in body frame components. Attitude control requires the angular position of body frame with respect to inertial reference frame. These angles may be described by three Euler angles. Euler angles should be used with care for angles $\geqslant 90^{\circ}$, but perform well for small angles and are chosen in the following simulations. If the Euler angles $\phi, \theta, \psi$ are small in magnitude, the relationship between body angular velocities and Euler angular velocities may be approximated as (Bryson, 1994),

$$
\begin{aligned}
& \omega_{x} \cong \dot{\phi}-\psi \omega_{o} \\
& \omega_{y}=\dot{\theta}-\omega_{o} \\
& \omega_{z}=\dot{\psi}+\phi \omega_{o}
\end{aligned}
$$

\subsection{Gravity Gradient Torque}

A body with non-uniform mass-distribution will, when exposed to a quadratic decaying gravitational field, be influenced from a torque. For most spacecraft situations, the following simplifications can be made (Hughes, 1986).

- Only the gravitational field from the earth is considered.

- The satellite is small compared to its distance from the mass centre of the earth.

- The satellite consists of a single body.

The gravity gradient torque, using small Euler angle approximation and taking the principal axes as the reference axes, is given by (5), (Hughes, 1986),

$$
{ }^{B} T_{G}=-3 \omega_{O}^{2}\left[\begin{array}{c}
\left(I_{y}-I_{z}\right) \phi \\
\left(I_{x}-I_{z}\right) \theta \\
0
\end{array}\right]
$$

\subsection{Magnetic Control Torque}

A spacecraft exposed to a magnetic field will, supposing that the spacecraft has a magnetic coil, be influenced by a magnetic torque. Denoting the external magnetic 
flux density by $B$ and the magnetic moment by $m$, the torque acting on the spacecraft may be written

$$
{ }^{B} T={ }^{B} m \times{ }^{B} B={ }^{B} m \times\left(R_{B}^{o o} B\right)
$$

where: ${ }^{B} B$ is the magnetic flux density expressed in body frame, ${ }^{B} m$ is the magnetic moment resulting from a $\mathrm{N}$ turns air cored coil, ${ }^{\circ} B$ is the magnetic flux density expressed in orbit frame, $R_{B}^{o}$ is the rotation matrix from orbit to body. Controlling the magnetic moment ${ }^{B} m$ provides a mean of performing attitude control. The magnetic torque will according to (6) act perpendicularly to the magnetic moment vector ${ }^{B} m$ and the magnetic field vector ${ }^{B} B$. Three perpendicular electromagnetic coils are normally applied to fully control the satellite.

A model of the magnetic field of the earth should be established before the magnetic moment acting on the satellite can be determined. The magnetic field varies strongly at high altitudes. Lower altitudes, approximately $1200 \mathrm{~km}$ or less, have a relatively constant magnetic field. Hughes (1986) suggested using a dipole moment as the earth's magnetic field at low altitudes. Assuming that the rotation of the earth is much less than the angular velocity of the satellite, the satellite moves over the poles, the Euler angles are small and the rotation of the magnetic flux density, B, from orbit to body is ignored, due to small Euler angles, then the magnetic control torque may be expressed in body frame components as

$$
{ }^{B} T_{A}=B_{O}\left[\begin{array}{c}
2 m_{y} \sin \lambda_{m} \\
m_{2} \cos \lambda_{m}-2 m_{x} \sin \lambda_{m} \\
-m_{y} \cos \lambda_{m}
\end{array}\right]
$$

where: $\lambda_{m}$ is the latitude angle with respect to the geomagnetic equatorial plane and $\left[\begin{array}{lll}m_{x} & m_{y} & m_{z}\end{array}\right]^{T}$ is the magnetic moment expressed in body frame components.

\subsection{Complete Linearised Mathematical Model}

The rotational motion of the satellite including gravity gradient torque and magnetic coil torque written in body frame components becomes

$$
\begin{aligned}
& I_{x} \ddot{\phi}-4 \omega_{o}^{2}\left(I_{y}-I_{z}\right) \phi+\omega_{o}\left(I_{x}-I_{y}+I_{z}\right) \dot{\psi}+2 B_{O} m_{y} \sin \lambda_{m} \\
& I_{y} \ddot{\theta}=-3 \omega_{o}^{2}\left(I_{x}-I_{z}\right) \theta-2 B_{O} m_{x} \sin \lambda_{m}+B_{O} m_{z} \cos \lambda_{m} \\
& I_{z} \ddot{\psi}=-\omega_{O}^{2}\left(I_{y}-I_{x}\right) \psi-\omega_{O}\left(I_{x}-I_{y}+I_{z}\right) \dot{\Phi}-B_{o} m_{y} \cos \lambda_{m}
\end{aligned}
$$

If the states are given as

$$
x=\left[\begin{array}{llllll}
\phi & \dot{\phi} & \theta & \dot{\theta} & \psi & \psi
\end{array}\right]
$$

then (8) can be represented as a state-space model

$$
\left[\begin{array}{l}
\dot{x}_{1} \\
\dot{x}_{2} \\
\dot{x}_{3} \\
\dot{x}_{4} \\
\dot{x}_{5} \\
\dot{x}_{6}
\end{array}\right]
$$




$$
\begin{aligned}
& =\left[\begin{array}{cccccc}
0 & 1 & 0 & 0 & 0 & 0 \\
-\frac{4 \omega_{O}^{2}\left(I_{y}-I_{z}\right)}{I_{x}} & 0 & 0 & 0 & 0 & \frac{\omega_{O}\left(I_{x}-I_{y}+I_{z}\right)}{I_{x}} \\
0 & 0 & 0 & 1 & 0 & 0 \\
0 & 0 & -\frac{3 \omega_{O}^{2}\left(I_{x}-I_{z}\right)}{I_{y}} & 0 & 0 & 0 \\
0 & 0 & 0 & 0 & 0 & 1 \\
0 & -\frac{\omega_{O}\left(I_{x}-I_{y}+I_{z}\right)}{I_{z}} & 0 & 0-\frac{\omega_{O}^{2}\left(I_{y}-I_{x}\right)}{I_{z}} & 0
\end{array}\right] \\
& {\left[\begin{array}{l}
x_{1} \\
x_{2} \\
x_{3} \\
x_{4} \\
x_{5} \\
x_{6}
\end{array}\right]} \\
& +B_{O}\left[\begin{array}{ccc}
0 & 0 & 0 \\
0 & \frac{2 \sin \lambda_{m}(t)}{I_{x}} & 0 \\
0 & 0 & 0 \\
-\frac{2 \sin \lambda_{m}(t)}{I_{y}} & 0 & \frac{\cos \lambda_{m}(t)}{I_{y}} \\
0 & 0 & 0 \\
0 & -\frac{\cos \lambda_{m}(t)}{I_{z}} & 0
\end{array}\right]\left[\begin{array}{l}
m_{x} \\
m_{y} \\
m_{z}
\end{array}\right]
\end{aligned}
$$

\section{Control and Simulations}

The gravitational torque alone manages to stabilise the satellite, but the gravitational torque is essentially undamped, and accurate pointing requires additional control torques (Skullestad, 1999). The aim of the following simulations is to indicate what stability and pointing accuracy that may be achieved using gravity gradient stabilisation combined with fuzzy logic controlled magnetic coils. Table 1 lists the applied nominal satellite data.

Satellite height:

Moment of inertia about principal axes

(boom deployed)

Satellite orbit:

Gravity boom length

Tip mass:

Magnettorquers:
$1200 \mathrm{~km}$

$I_{x}=178 \mathrm{kgm}^{2}, I_{y}=181 \mathrm{kgm}^{2}, I_{z}=4.3 \mathrm{kgm}^{2}$ Polar orbit

$6 \mathrm{~m}$

$2 \mathrm{~kg}$

3 perpendicular magnetic coils. Each coil gives a magnetic moment of $8 \mathrm{Am}^{2}$

Table 1. Applied satellite data. 
Three-axis attitude control requires attitude angles and also attitude angular rate information. A star sensor and a 3-axes magnetometer combined in a Kalman filter provide sufficient accurate roll, pitch and azimuth angle and angular rate information (Kyrkjebø, 2000).

The movements of the satellite are large shortly after release from the launch vehicle. This paper assumes that the control system successfully has performed a detumbling operation, and concentrate on the attitude accuracy that can be achieved during small angle deviations, i.e. in normal space flight.

The dynamic equations of the satellite are non-linear, coupled and have multiple inputs and outputs. If, however, the simulations are limited to small angle deviations, i.e. after the detumbling phase has ended, the linearized mathematical models presented in section 3 may successfully be applied. The satellite is released straight above equator in all simulations.

\subsection{The Fuzzy Controller}

The process, shown in (8), is time-varying, multivariable, coupled and the control action is time-varying. The control torques, using magnetic coils, can only be generated perpendicular to the local magnetic field vector, and the roll axis become non-controllable over equator and the azimuth axis become non-controllable over the poles. This can easily be seen from the B matrix in (9), $\lambda_{m}$ is zero over equator and $90^{\circ}$ over the poles. Roll and azimuth are coupled as seen from the A matrix and both axes are controlled from the y-coil, as seen from the B matrix. Facing this process it becomes difficult to achieve accurate attitude control simultaneously in both roll and azimuth. The pitch axis is, however, decoupled from both roll and azimuth and is easily controlled. The pitch controller is often, also in this paper, developed separately from the roll and the azimuth controllers.

The MATLAB Fuzzy Logic Toolbox was used to build the fuzzy controller. Figure 3 shows the fuzzy controller for all three axes.

A single controller (fis function) is used to control the pitch axis, named "Fuzzy Logic Controller" in Figure 3. For the roll and azimuth axis there are three different controllers built as S-functions, named "xzaksefis" in Figure 3. The S-functions are programmed as script files in MATLAB. This gives the opportunity to change the membership functions and parameters for the controllers in real time and makes the system adaptive. The satellite latitude angle (with respect to the geomagnetic equator-

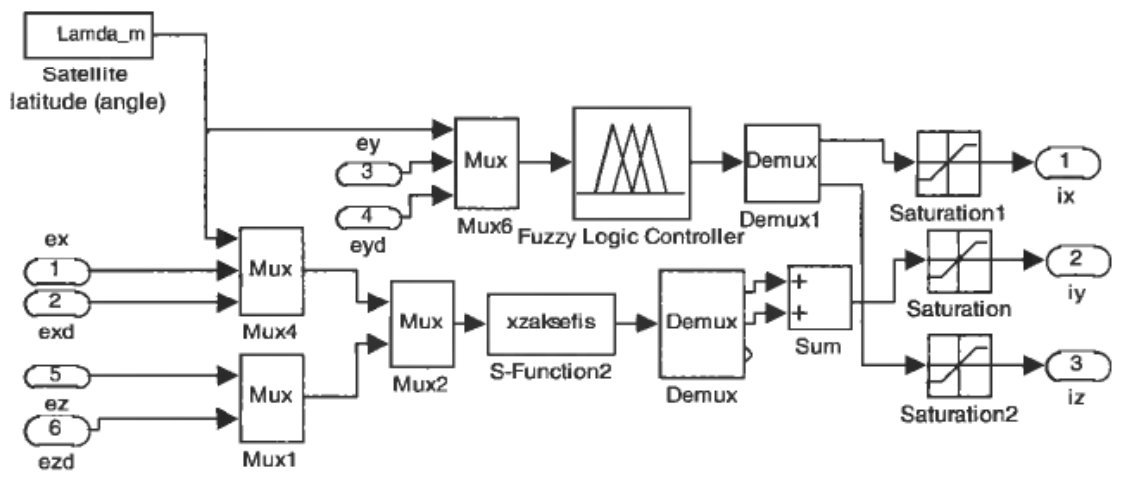

Figure 3. Fuzzy Logic Controller for all three axis. 


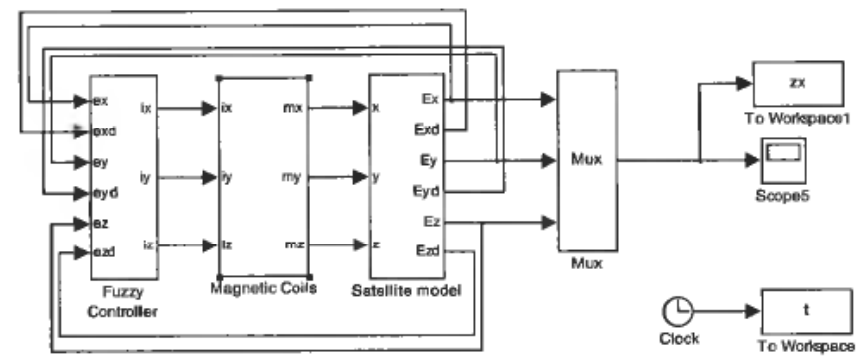

Figure 4. Complete Simulink model.

ial plane), the Euler angles, ex, ey, ez, and the Euler angle velocities, exd, eyd, ezd, are inputs to the controllers and the coil currents, ix, iy, iz, the are outputs. Saturation blocks are used to avoid currents above $0.25 \mathrm{~mA}$, which is the upper limit for the available coil currents.

Figure 4 shows the complete Simulink model of the satellite, including the fuzzy logic controllers. The subsystem named "Fuzzy Controller" contains the controllers shown in Figure 3. A mathematical model of the magnetic coil moment is modelled into the subsystem named "Magnetic Coils" and the subsystem named "Satellite Model" contains the mathematical model of the satellite, as documented in (9).

\subsection{Pitch controller}

The pitch controller is based on 4 membership functions and 60 rules. The satellite model shown in Figure 4 is simulated using initial conditions imposed on the Euler angle around the y-axis, only. Figure 5 shows the fuzzy logic controlled Euler angle, $\theta$, versus time. The initial condition is given as $[\theta]=\left[10^{\circ}\right]$. One orbital period is 6545 sec. The pitch angle error is reduced to $0.2^{\circ}$ after 0.3 orbital periods. The pitch angle error becomes less than $10^{-2 \circ}$ after 1.5 orbital periods, but unmodelled disturbances, like residual magnetic moments, variations in the magnetic field of the earth etc. are expected to worsen the angle accuracy. More accurate models indicate pitch error in the range of $0.1-0.5^{\circ}$.

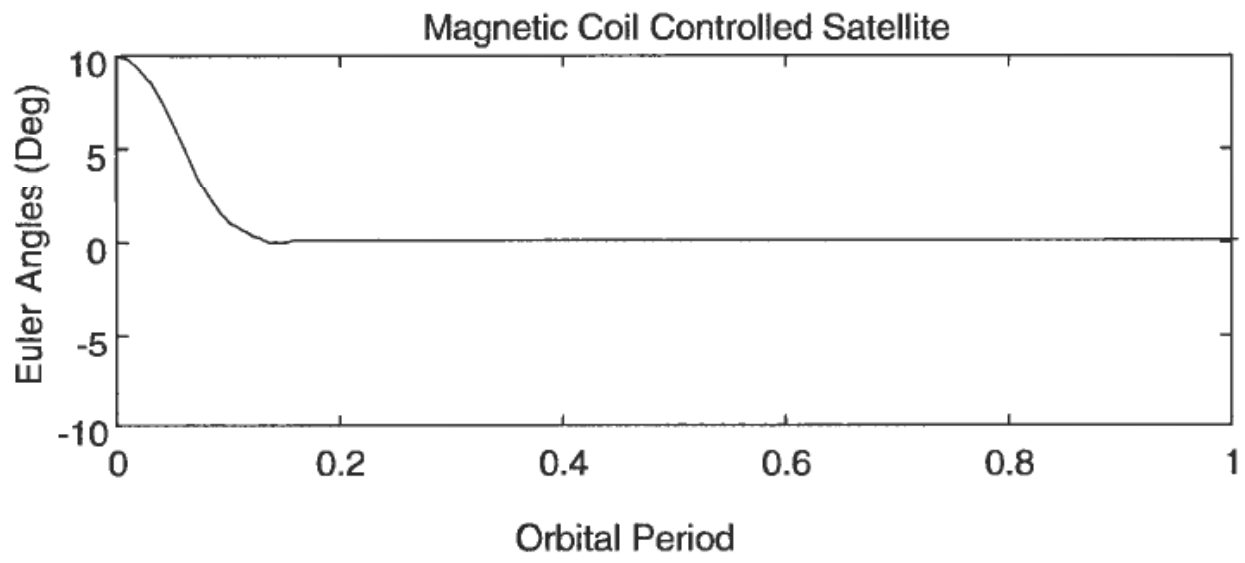

Figure 5. Euler angle, $\theta$, versus time for a fuzzy logic controlled satellite, exposed to an initial angle of $10^{\circ}$. 


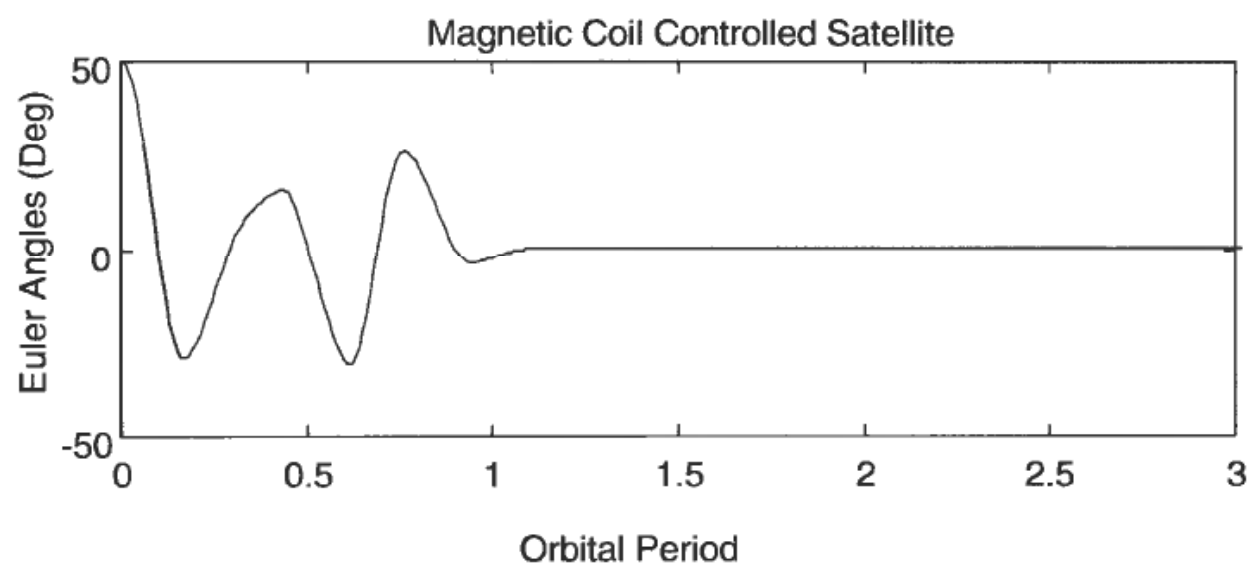

Figure 6. Euler angle, $\theta$, versus time for a fuzzy logic controlled satellite, exposed to an initial angle of $50^{\circ}$.

The fuzzy controller controls the pitch axis quite satisfactory using an initial angle of $10^{\circ}$. A new simulation is performed, but this time with an initial pitch angle of $50^{\circ}$. Figure 6 shows the fuzzy logic controlled Euler angle, $\theta$, versus time. The initial condition is given as $[\theta]=\left[50^{\circ}\right]$. This time the fuzzy logic controller needs more time to stabilise the rotation about the y-axis, but the pitch angle error becomes less than $0.5^{\circ}$ after 1.7 orbital periods. The pitch angle error is, as for the $10^{\circ}$ initial condition, reduced to less than $10^{-20}$ after 3 orbital periods. An initial angle of $50^{\circ}$ stretches the linearised model assumptions, and changes in the response behaviour will be expected, using a non-linear satellite model.

The moment of inertia of the $y$-axis is varied $\pm 10 \%$, the applied model uncertainty makes small changes in the pitch behaviour. A small disturbance impulse torque is imposed in pitch after the $y$-axis is stabilised to a pith error of $< \pm 1^{\circ}$. The $y$-axis rotate, due to the imposed disturbance torque, but not more than expected, and the pitch angle error is back to $<10^{-20}$ after a few orbital periods, depending on the size of the disturbance.

The magnetic coils wrapped around the $\mathrm{x}$ and $\mathrm{z}$-axes both control the $\mathrm{y}$-axis and it may be possible to remove one magnetic coil and still be able to control the y-axis. The z-coil was removed. Simulations show only small changes in the pitch response compared to the results obtained using 2 coils, but the y-axis will have less control torque available.

\subsection{Roll and Azimuth Controller}

The roll and azimuth axes are both controlled from the y-coil, and the control strategy become much more difficult. A possible approach was to minimise the cross coupling between the axes and then separately design a controller for each axis. The controllers are activated or deactivated depending on the latitude angle, where the $\mathrm{x}$ axis is mainly controlled over the poles, whilst the z-axis is mainly controlled over the equator. A way of keeping the cross coupling low is to keep the angular velocity of both the $\mathrm{x}$ and $\mathrm{z}$-axes low.

The chosen control strategy gives an effectively and nearly linear control action affecting one axis at a time. The roll axis is controlled from one controller using 5 


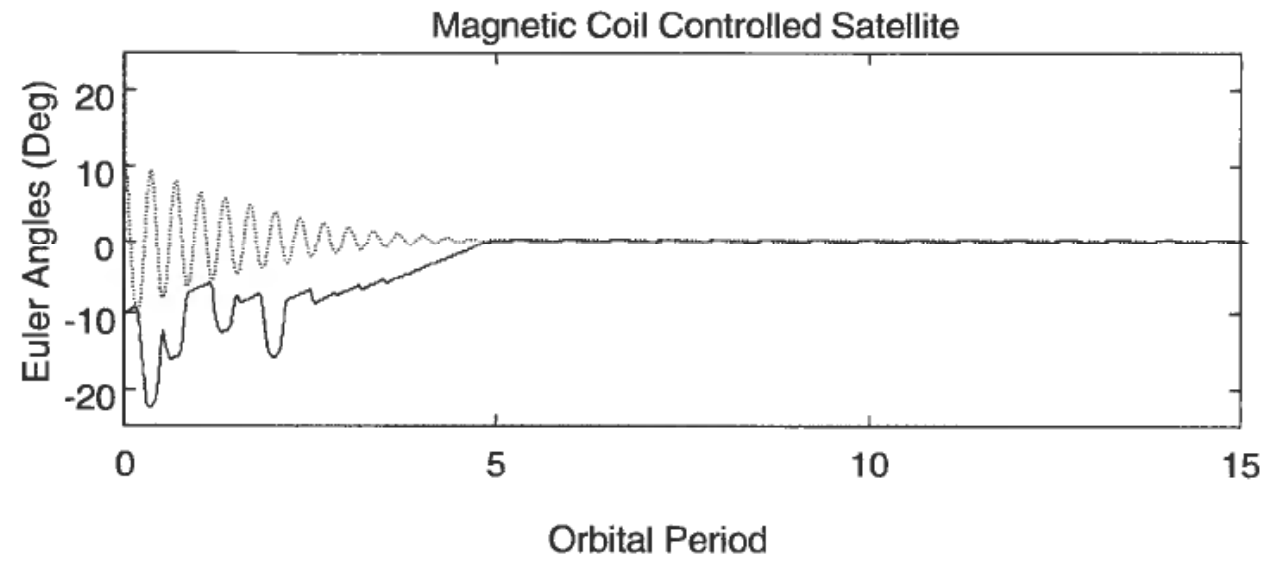

Figure 7. Euler angles, $\varphi(\cdots)$ and $\psi(-)$, versus time for a fuzzy logic controlled satellite, exposed to an initial angle of $10^{\circ}$ in both axes.

membership functions and 33 rules. The azimuth axis is controlled from two controllers, one for large angular errors and one for small angular errors. The large angular error controller uses 5 membership functions and 47 rules, whilst the small angular error controller uses 7 membership functions and 30 rules.

The complete mathematical model shown in Figure 4 is simulated using initial conditions imposed on the Euler angles around the $\mathrm{x}$ and z-axis, only. Figure 7 shows the fuzzy logic controlled Euler angle, $\varphi$ and $\psi$ versus time, the initial conditions are given as $[\varphi \psi]=\left[10^{\circ}, 10^{\circ}\right]$. Roll angle $=$ dotted line, azimuth angle $=$ solid line. The $\mathrm{x}-$ axis is controlled to an roll angle error of less than $0.2^{\circ}$ and the z-axis to an azimuth angle error of less than $\pm 0.1^{\circ}$ after approximately 8 orbital periods. Both the azimuth and roll angle error become less than $0.1^{\circ}$ after 16 orbital periods, but disturbances is as explained in section 4.2, expected to worsen the result, and errors of $0.5-1.0^{\circ}$ may be a reality.

The fuzzy logic controllers manage to control the roll and azimuth angles accurately using initial conditions of $10^{\circ}$. Figure 8 shows the fuzzy logic controlled

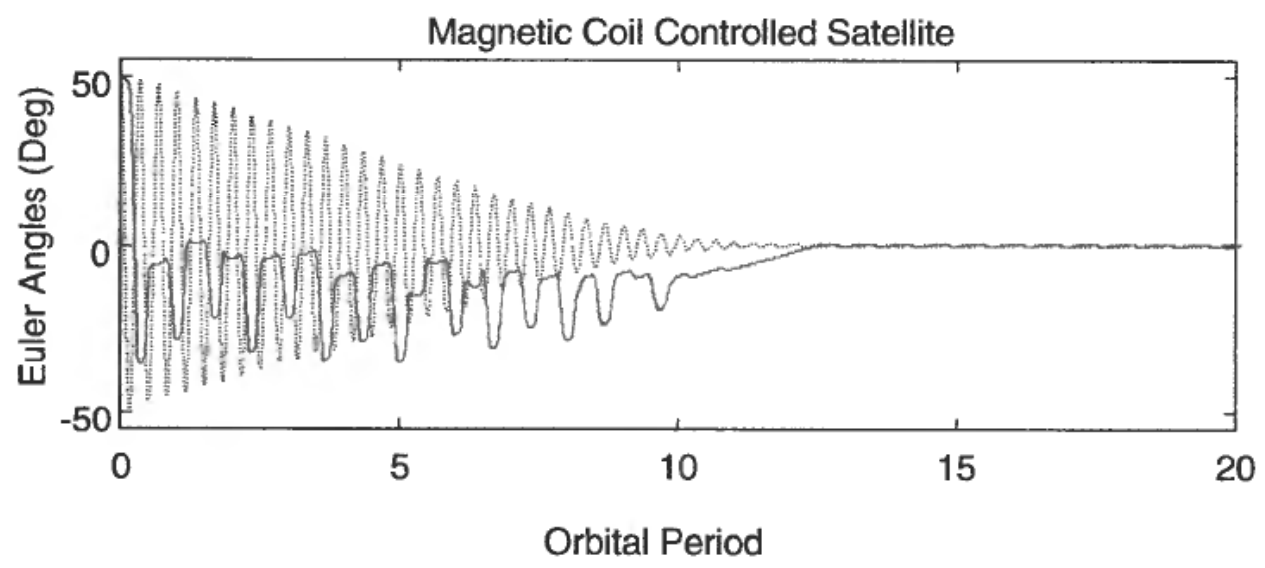

Figure 8. Euler angles, $\varphi(\cdots)$ and $\psi(-)$, versus time for a fuzzy logic controlled satellite, exposed to an initial angle of $50^{\circ}$ in both axes. 
Euler angles, $\varphi$ and $\psi$ versus time, the initial conditions is given as $[\varphi \psi]=\left[50^{\circ} 50^{\circ}\right]$. These larger initial conditions force the controllers to work harder in order to stabilise the roll and azimuth axes, but the axes are accurately controlled, even if the settling time become longer. Both axes are controlled to within $0.2^{\circ}$ after 18 orbital periods, and $0.1^{\circ}$ after 30 orbital periods. Non-linear models may, as explained in section 4.2, be a better choice when using large initial conditions.

The roll axis has a moment of inertia of $181 \mathrm{kgm}^{2}$, whilst the azimuth axis has a moment of inertia of $4.3 \mathrm{kgm}^{2}$. A strong roll control easily swings the azimuth axis away from zero and the control actions should be carefully imposed. On the other hand, a careful control action slows down the roll response. The aim is to compromise. The roll gain may look high and lowering the roll gain will reduce the roll angle ringing, but at the cost of a slower control action. Increasing the moment of inertia in azimuth may simplify the control task.

The moment of inertia in roll and azimuth is changed $\pm 10 \%$ in turn, these model uncertainties influencing little on the satellite response. Neither do imposed impulse torque worsen the azimuth and roll angle errors.

\section{Conclusion}

This paper describes control of a small gravity gradient stabilised satellite using fuzzy logic controlled magnetic coils. The y-axis is de-coupled the $\mathrm{x}$ - and $\mathrm{z}$-axes and the pitch controller was designed separately from the roll and azimuth controllers. The $y$-axis is controlled from both the $\mathrm{x}$-coil and z-coil and was relatively easy to design. The chosen pitch controller applies 4 membership functions and 60 rules. The controller gives an excellent pitch angle error of $0.2^{\circ}$ after 0.3 orbital periods, and less than $10^{-20}$ after 3 orbital periods, but it is expected that disturbances like residual magnetic moment in the satellite, variations in the magnetic field of the earth, air drag etc. will worsen the result.

Simulations show that it is possible to remove one coil and still obtain acceptable stability and pointing accuracy, but at the cost of less robustness. Removing one coil may allow a cheaper satellite solution.

The $\mathrm{x}$ and $\mathrm{z}$-axis is strongly coupled and both axes are be controlled from one common magnetic coil, i.e. the y-coil. Designing accurate roll/azimuth controllers are a difficult challenge. The control strategy was to minimise the cross coupling between the $\mathrm{x}$ and $\mathrm{z}$-axes, by penalising the angular velocities in the respective axes. This strategy makes it possible to design the roll and azimuth controller separately, but at the cost of a relatively slow control action.

The roll controller applies 5 membership functions and 33 rules. The azimuth controller is made adaptive with regards to the azimuth angular error. One controller is designed for small angular errors and applies 7 membership functions and 30 rules. One controller is designed for large angular errors and applies 5 membership functions and 47 rules. The roll and azimuth controllers achieve a pointing accuracy of $0.2^{\circ}$ after 18 orbital periods, and $0.1^{\circ}$ after 30 orbital periods. As for control of the $y$ axis, disturbances like residual magnetic moment in the satellite, variations in the magnetic field of the earth, air drag etc. is expected to worsen the azimuth and roll angle error.

The main problem with the combined magnetic torquing and gravity control is that the available torque is limited to being orthogonal to whatever is the local B vector. 


\section{References}

Aaström, K. J. and Wittenmark, B. (1984). Computer Controlled Systems. Prentice Hall, Englewood Cliffs, N.J.

BAK, T., WiSNIEWSKI, R. and Blanke, M. (1996). Autonomuous Attitude Determination and Control System for the Ørsted Satellite. In proc.: IEEE Aerospace Application Conference.

Byrnes, C. I. and Isidori, A. (1991). On the Attitude Stabilization of Rigid Spacecraft. Automatica, Vol. 27, No. 1, pp. 87-95.

Bryson, A. E. (1994). Control of Spacecraft and Aircraft. Princeton University Press, New Jersey.

Cavallo, A., De Maria, G., Ferrara, F. and Nistri, P. (1993). A Sliding Manifold Approach to Satellite Attitude Control. In proc.: $12^{\text {th }}$ World Congress IFAC, Sidney.

Green, M. and Limebeer, D. J. N. (1995). Linear Robust Control. Prentice Hall, Englewood Cliffs, N.J.

Hughes, P. C. (1986). Spacecraft dynamics, John Wiley \& Sons, Inc.

KANE, T. K, Likins, P. W. and Levinson, D. A. (1983). Spacecraft Dynamics. McGraw-Hill, Inc

Kaplan, M. H. (1976). Modern Spacecraft Dynamics and Control. John Wiley, New York.

KYRKJEBØ, E. (2000). Satellite Attitude Determination. M.Sc. Thesis, NTNU.

Musser, K. L. and Ebert, W. L. (1989). Autonomuous Spacecraft Attitude Control using Magnetic Torquing Only. In proc.: Flight Mechanics Estimation Theory Symposium, NASA.

NARHEIM, B. T. and SveneS, K. R. (1994). Norwegian Ionospheric Small Satellite Experiment (NISSE). Phase-A Study Report.

Passino, K. M. and Yukovich, S. (1998). Fuzzy Control. Addison-Wesley

RAO, S. (1990). Mechanical Vibrations. Addison Wesley.

Skogestad, S. and Postlethwaite, I. (1996). Multivariable Feedback Control Analysis and Design. Wiley

SKULleSTAD, Å. (1995). Identification of vibration parameters in a space structure. PhD Thesis University of Oslo.

SkullestaD, A. (1999). Modelling and Control of a Gravity Gradient Stabilised Satellite. Modeling, Identification and Control, Vol. 20, No. 1.

WisNiEwSKI, R. (1996). Satellite Attitude Control Using Only Electromagnetic Actuation. $\mathrm{PhD}$. Thesis. University of Aalborg.

ZADEH, L. A. (1965). Fuzzy sets. Informant. Control. 8. 